\title{
Cloud Computing in SMEs: A Qualitative Approach to Identify and Evaluate Influential Factors
}

\author{
Gregor Friedrich-Baasner \\ University of Wuerzburg \\ gregor.friedrich-baasner@uni- \\ wuerzburg.de
}

\author{
Marcus Fischer \\ University of Wuerzburg \\ marcus.fischer@uni- \\ wuerzburg.de
}

\author{
Axel Winkelmann \\ University of Wuerzburg \\ axel.winkelmann@uni- \\ wuerzburg.de
}

\begin{abstract}
In the age of ubiquitous digitalization, there are opportunities to relocate miscellaneous activities to the cloud, e.g. data storage, computing operations or even entire services. This has a massive impact on nearly all branches of industries as well as for private individuals. However, the reasons for adoption or rejection of these technologies are still underrepresented in academic literature.

During the study at hand, qualitative expert interviews are designed and conducted to elicit reasons for the adoption of cloud services in German small and medium-sized enterprises (SME).

The study presents a list of explored influencing factors, ranked in order to their relevance. In addition to the ones abstracted from the literature, the study identifies five more factors with an influence on the adoption of cloud services in the investigated area. The result of the evaluation is that the security and handling of data have the highest significance for German SMEs. Thus, trust, privacy and security are the most relevant influencing factors.
\end{abstract}

\section{Introduction}

Adoption and diffusion are key success factors for every innovation. The adaptors' benefits are often assumed as a crucial fact for novel technologies and their diffusion. In most cases, the diffusion of innovations is a rather slow process [27]. This appears to be true for the dissemination of cloud services within the SME sector as well. Many different factors are usually influencing the adoption in both directions, accelerating and inhibiting [13]. These factors differ in their origin, from the technology, over the organization to some from the environmental background.

Despite the obvious opportunities created through the usage of cloud services, the acceptance and diffusion are low, especially among SMEs. A recent survey within German companies shows that only 44 percent are currently using cloud services. But the interest in cloud service solutions is rising and for the first time the amount of supporters overreached the sceptics [13].

Due to this divergence between opportunities and resentments, this study was designed to examine the factors influencing the acceptance and adoption of cloud services.

There are a variety of definitions of cloud sevices. This paper based on the NIST definition of cloud computing by Mell and Grance [20].

The usage of cloud services provides new opportunities for decisive competitive advantages, especially for SMEs. For example, the billing method pay per use allows to calculate the costs considering the resources that were actually used. The usually accruing high initial investment could be circumvented that way. Therefore, released capacities and resources can be utilized for the core business and the advancement of the enterprise. Through the rental of external resources, new additional opportunities arise that were previously not possible because of unfeasible investments. Cloud services allow SMEs the usage of resources formerly exclusively used by big companies with own data centers [19]. Furthermore, Schneider and Suyaev stated that there is a need of empirical clarified reasons for cloud service adoption [30].

The above mentioned situation motivated this research to investigate factors influencing the acceptance and adoption of cloud services within organizations. Furthermore, SMEs play a significant role in the economy. The European Union defined several criteria for SMEs to be segmented as such. One of the criteria is the size of the company which is limited by 250 employees. Another one is annual turnover which must not exceed 50 million euro [7]. In 2015, 99.3 percent of German companies were categorized as SME. These companies took responsibility for around 60 percent of the entire German employment [32]. For these reasons, this 
research focuses on the acceptance and adoption of cloud services in SMEs.

The objective of this paper is to identify and to evaluate factors influencing the adoption of cloud services. The already mentioned positive effects on entrepreneurial success through the integrated application of cloud services provides SMEs the chance to keep up with larger companies in a highly competitive global market.

The following research question (RQ) is assessed by this paper:

RQ: Which factors are influencing German SMEs in the decision to adopt or not adopt cloud services?

The remainder of this paper is organized as follows. Section 2 provides an overview of the existing studies in this area. Influencing factors for the acceptance and adoption from these studies were consolidated and evaluated within this chapter. The third section presents the research methodology used to answer the posed research question. This chapter is subdivided into an overview of the conducted expert interviews and the subsequent content analysis. Section 4 deals with the results of the content analysis and details on the empirical survey. This is followed by a discussion of the identified influence factors. The final conclusion, limitations and remarks on future research are combined in chapter 6 .

\section{Related Work}

The systematic review of relevant literature provides an overview of studies in a certain field and identifies areas with potential for further investigation. The literature review also provides an outline of already investigated factors [36].

This study is based on a literature analysis conducted according to the framework proposed by vom Brocke et al. [4].

In order to identify publications concerning the research question, we used the database SCOPUS with the keywords cloud, adoption and acceptance. There are two wide ranged literature reviews [11, 30] on which the factors in table 1 and the conducted literature review in this paper is founded on.

Because of the already existing literature reviews [30] the paper on hand proceeds the search from 2015 till 2017 and identified additional 117 contributions. Following a rigour full-text analysis, 29 of the articles were identified as relevant. Furthermore, a forward and backward search results to 10 more high cited papers. Examples of the total 39 relevant papers are shown in table 1 to provide an overview of the influencing factors extracted from the relevant literature.

The factors in table 1 are grouped in four categories, individual, organizational, technological and environmental. In accordance with the particular category, each corresponding factor was categorized to give a better oversight. The categories are derived from the technology-organizational-environmental framework by Tornatzky and Fleischer [34]. In addition, the categories are extended by the impact on individuals obtained from Venkatesh and Davis [35].

In the course of the literature analysis, 33 factors were identified which were considered as relevant regarding acceptance and adoption of cloud services. The mapping of the factors (see Table 1) is based on previous studies [11, 30].

Table 1: Cluster of influencing factors

\begin{tabular}{|c|c|c|c|}
\hline Technological & Organizational & Environmental & Individual \\
\hline $\begin{array}{l}\text { Deferral } \\
\text { Options } \\
{[11,30]}\end{array}$ & $\begin{array}{c}\text { External Know- } \\
\text { How } \\
{[11,16,30]}\end{array}$ & $\begin{array}{c}\begin{array}{c}\text { Subjective } \\
\text { Norm }\end{array} \\
{[11,30,37]}\end{array}$ & $\begin{array}{c}\text { Trust } \\
{[10,15,30]}\end{array}$ \\
\hline $\begin{array}{c}\text { Asset Specify } \\
{[3,11,30]}\end{array}$ & $\begin{array}{c}\text { Roles and } \\
\text { Responsibility } \\
{[3,11,30]}\end{array}$ & $\begin{array}{c}\text { Industry } \\
\text { Characteristics } \\
{[1,18,30]} \\
\end{array}$ & $\begin{array}{c}\text { Perceived } \\
\text { Usefulness } \\
{[12,30,31]}\end{array}$ \\
\hline $\begin{array}{c}\text { Capital Costs } \\
{[10,11,30]}\end{array}$ & $\begin{array}{c}\text { Lock-in-Effect } \\
{[11,23,30]}\end{array}$ & $\begin{array}{l}\text { Privacy } \\
{[8,10,11]}\end{array}$ & $\begin{array}{c}\text { Perceived } \\
\text { Ease of Use } \\
{[9,29,30]}\end{array}$ \\
\hline $\begin{array}{c}\text { Cost Reduction } \\
{[11,24,30]}\end{array}$ & $\begin{array}{c}\text { Organization } \\
\text { Size } \\
{[1,11,30]} \\
\end{array}$ & $\begin{array}{c}\text { Competitive } \\
\text { Pressure } \\
{[12,24,30]}\end{array}$ & \\
\hline $\begin{array}{c}\text { Growth Options } \\
{[11,21,30]}\end{array}$ & $\begin{array}{c}\text { Usage } \\
\text { Frequency } \\
{[11,30,37]}\end{array}$ & $\begin{array}{c}\text { Social Influence } \\
{[15,28,30]}\end{array}$ & \\
\hline $\begin{array}{c}\text { Uniqueness } \\
{[11,23]}\end{array}$ & $\begin{array}{l}\text { Complexity } \\
{[9,24,30]}\end{array}$ & $\begin{array}{c}\text { Energy } \\
\text { Efficiency } \\
{[21]}\end{array}$ & \\
\hline $\begin{array}{l}\text { IT Flexibility } \\
{[11,17,30]}\end{array}$ & $\begin{array}{c}\text { Effort } \\
\text { Expectation } \\
{[2,15]}\end{array}$ & $\begin{array}{c}\text { Image } \\
{[23,26,30]}\end{array}$ & \\
\hline $\begin{array}{c}\text { Security } \\
{[3,11,30]}\end{array}$ & $\begin{array}{c}\text { Voluntariness } \\
\text { of Use } \\
{[9,23,30]}\end{array}$ & & \\
\hline $\begin{array}{c}\text { Strategic } \\
\text { Impact } \\
{[11,22,30]} \\
\end{array}$ & $\begin{array}{c}\text { Service } \\
\text { Monitoring } \\
{[21,26,30]} \\
\end{array}$ & & \\
\hline $\begin{array}{c}\text { Technological } \\
\text { Availability } \\
{[3,26,30]} \\
\end{array}$ & $\begin{array}{c}\text { Compatibility } \\
{[9,24,30]}\end{array}$ & & \\
\hline & $\begin{array}{l}\text { Trialability } \\
{[1,9,17]}\end{array}$ & & \\
\hline & $\begin{array}{c}\text { Relative } \\
\text { Advantage } \\
{[16,24,30]}\end{array}$ & & \\
\hline & $\begin{array}{c}\text { Service } \\
\text { Controlling } \\
{[14,17,30]}\end{array}$ & & \\
\hline
\end{tabular}

The objective of the study at hand is to verify or falsify the factors identified in previous studies and, if necessary, to add new ones that have not been identified in other research so far. Hence, table 1 serves as foundation for the following empirical examination.

\section{Methodology}

The goal of the study is to investigate factors influencing the adoption of cloud services, specifically 
in German SMEs. Hence, the factors identified in chapter 2 need to be matched with appropriate data collected from the target group. Furthermore, we aim to explore additional factors that might not have been identified in previous studies. In order to simultaneously gather data of confirmative and exploratory character, we applied a qualitative approach. Hence, the study on hand follows the established procedure of qualitative research as proposed by Bryman and Bell [5]. Figure 1 shows the six basic steps necessary to perform this kind of analysis, whereas steps 1-3 relate to the data collection process and steps 4-6 describe the data interpretation process.

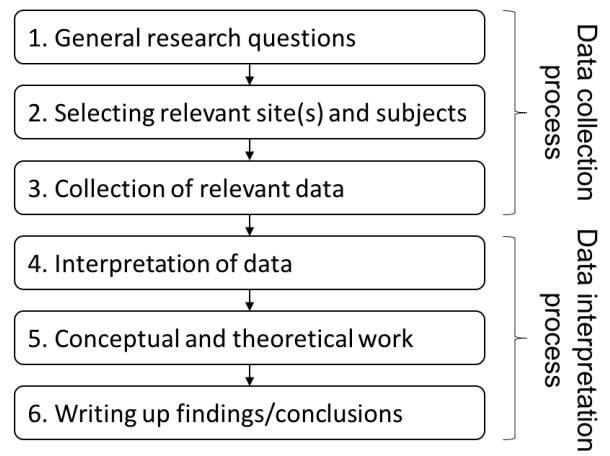

Figure 1. Main steps of qualitative research [5]

\subsection{Data collection process}

As figure 1 shows, the data collection process starts with the statement of an explicit research question. Within the scope of this paper, the research question has been stated before as follows: Which factors are influencing German SMEs in the decision to adopt or not adopt cloud services?

In order to answer this question, appropriate data from the target group need to be collected and analyzed regarding the influence of the factors derived from the publications examined in chapter 2 and the appearance of effects not identified so far. For the purpose of collecting the right data, a suitable collection method needs to be chosen and the target group needs to be defined precisely by selecting relevant subjects in step 2. As for the collection method, expert interviews proved to be the right instrument to gather data that can be matched with the coding scheme derived from existing factors on the one hand and examined regarding unknown effects on the other hand [25]. The selection of experts was made by applying the following criteria: interviewees had to (1) have comprehensive knowledge of information technology and cloud services in particular, (2) be employed by a German SME using or providing cloud services, and (3) be in a job position that allows to be responsible for or have a say in strategic IT sourcing decisions. Based on these criteria, interviews were executed with the fifteen participants shown in table 2. P1 to P3 represents the participants of the pretest which were not included in the results of the survey and I1 to I12 the interviewees.

Table 2. Sample overview

\begin{tabular}{|c|c|c|}
\hline Number & Industry & $\begin{array}{c}\text { Number of } \\
\text { employees }\end{array}$ \\
\hline P1 & Business Information Systems & \\
\hline P2 & Jurisprudence & \\
\hline P3 & Business Information Systems & \\
\hline I1 & IT development & $<10$ \\
\hline I2 & IT service/healthcare & $<10$ \\
\hline I3 & IT service & $<50$ \\
\hline I4 & e-commerce & $<10$ \\
\hline I5 & IT development/service & $<50$ \\
\hline I6 & Law firm & $<10$ \\
\hline I7 & IT development/service & $<250$ \\
\hline I8 & Fintech & $<10$ \\
\hline I9 & IT service & $<50$ \\
\hline I10 & IT service & $<250$ \\
\hline I11 & High-tech production & $<10$ \\
\hline I12 & IT service/healthcare & $<15$ \\
\hline Affterwards the data collection process & \\
\hline
\end{tabular}

Afterwards, the data collection process ends with the actual collection of relevant data in step 3 . This includes a clear documentation of the development of a target-aimed interview guide, the administrative work of coordinating and performing appointments with interviewees as well as the preparation of data [6]. The semi-structured interview guide focused on open questions regarding drivers and barriers of cloud adoption from different perspectives, e.g. technological or juridical. Thus, the interviewee was not directed or limited in his answers in any way by having knowledge of the list of already known influence factors. The guide was tested and slightly adjusted by performing three pretest interviews with academic and nonacademic professionals in the field of IT. Twelve expert interviews were conducted personally or by phone in the main study, which took place from September 2015 until January 2016. All interviews were recorded upon approval by the interviewee and fully transcribed afterwards. Eventually, the call $\operatorname{logs}$ were sent to each participant for confirmation in order to ensure the content validity [5].

\subsection{Data interpretation process}

The data interpretation process contains three steps: the interpretation of data, the conceptual and theoretical work, and the composition of the results. First, the collected and transcribed data need to be analyzed and interpreted in order to gain actual information from the raw material. As for the study on hand, this means that the interview protocols needed to be matched with the coding scheme derived from the 
results of the literature analysis shown in table 1 . Furthermore, the protocols had to be examined particularly with regard to adoption criteria that have not been captured by previous studies. For that purpose, we performed a comprehensive content analysis [25]. In order to ensure reliability, the examination was undertaken by three researchers in a double-blind process using the data analysis software $M A X Q D A$ in release 12.1.3. Afterwards, the results were matched and, if discrepancies occurred in respect of the assignment between factors and text passages, harmonized by majority voting.

In step 5, the results from the coding process need to be further processed and used for theoretical and conceptual work. We decided to apply the established TOE-framework to further categorize the evaluated as well as the anew identified influence factors [34]. Figure 2 shows the theoretical framework which contains the three perspectives technology, organization, and environment. In the study on hand, the framework was extended by the perspective individual in order to represent factors that could not reasonably be assigned to the primary perspectives.

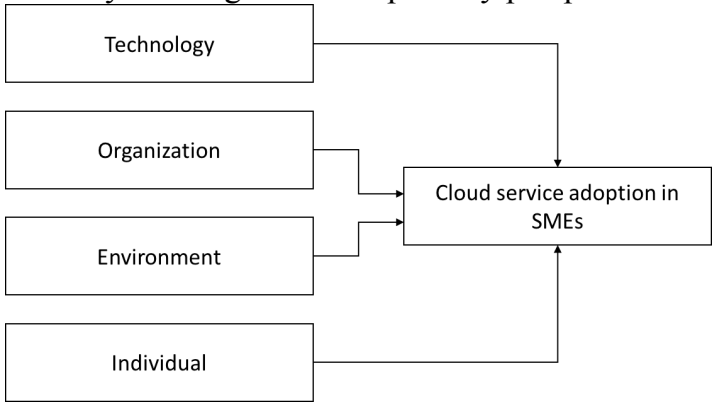

Figure 2. Theoretical framework [22]

In addition to the evaluation, exploration and categorization of influence factors, a frequency analysis was performed to better understand and visualize the weightings of the factors in the field on the basis of their denominations [38]. The results of these analyzes are presented subsequently in chapter 4 .

Eventually, step 6 of the procedure model for qualitative analysis (see fig. 1) describes the structured composition of the study results in the form of an article or work report, which is represented by the paper at hand.

\section{Findings}

In order to generate empirical evidence from the qualitative data regarding the weightings of the influence factors, we performed a frequency analysis using a coding scheme derived from the factors characterized in previous studies (see figure 3 ).

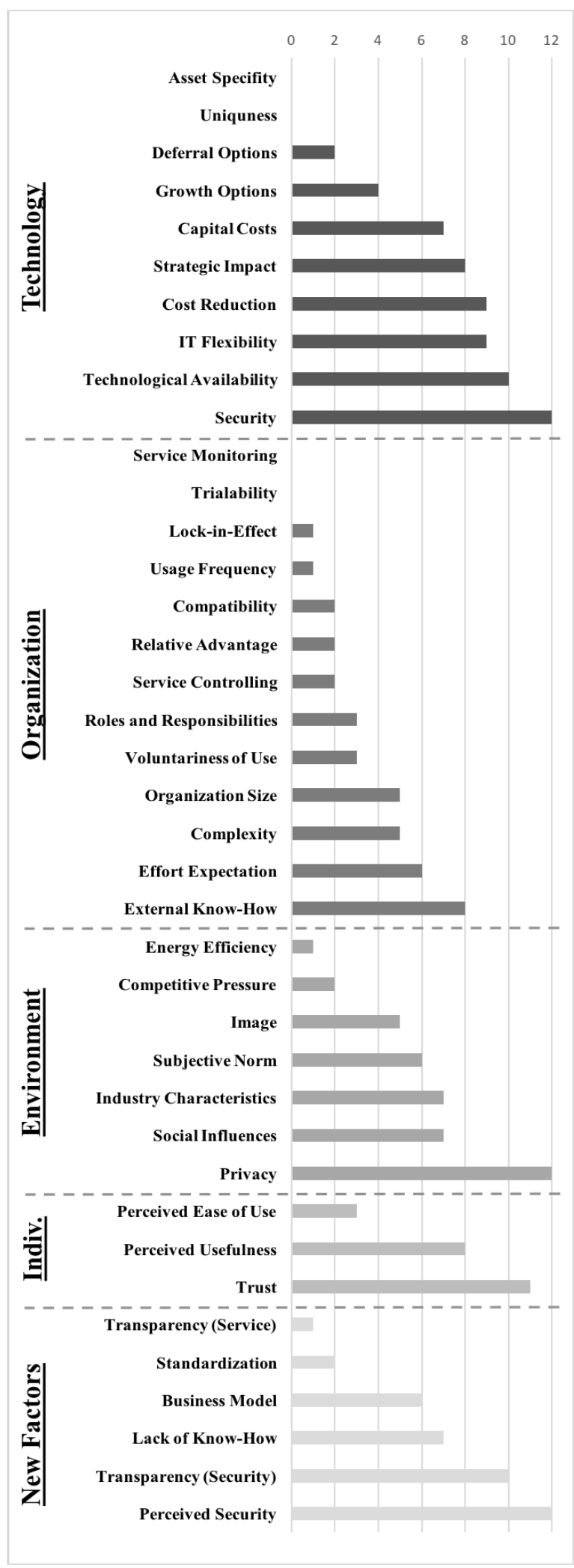

Figure 3. Findings

Initially, we assigned each factor to one of the four perspectives of the TOEI-framework (see table 1) 
following the approach of Stieninger and Nedbal [33]. Afterwards, this coding scheme composed of codes (perspectives) and subcodes (factors) was incorporated into the qualitative data analysis software MAXQDA. Subsequently, three researchers independently examined the interview protocols matching the respective expert statements with the subcodes of the coding scheme. The final results of this procedure are shown in figure 3 , whereas the bars represent the number of experts who emphasized the influence of the particular factor on their decision to adopt or not adopt cloud services in their company. Detailed information with references to selected interviewee statements for each perspective are provided in the following subchapters. As far as linguistically possible, quotes were translated into English without any alterations.

\subsection{Technological perspective}

The technological perspective contains ten influence factors, whereof the following eight could be verified in the German SME sector: security, technological availability, IT flexibility, cost reduction, strategic impact, capital costs, growth options, and deferral options. The factors asset specifity and uniqueness, which could be proved in previous studies, were not emphasized by any of the experts in our sample.

The factor security was mentioned by all interview partners $(n=12)$. In contrast to other examinations in the field, experts from German SMEs do not only assess this criterion negatively. Interviewee (I4) states: "The data security [of cloud-services] is much higher than the data security of internal systems". Expert (I3) affirmed this picture: "Security concepts are a basic module and essential service for every cloud provider. I think cloud providers are already well prepared because of the architecture itself and the privacy requirements". However, some experts still make reservations regarding the security of cloud-based IT services: "If someone wants to compromise us [...], he is pretty likely to be able. And if [the system] is in the cloud, he usually has access to different accounts immediately" (I7).

The second most frequently mentioned technological factor is technological availability $(\mathrm{n}=$ 10). With regard to the the cloud-readiness in Germany, all interviewees agree that "the infrastructure in Germany can and has to be improved" (I3).

IT flexibility was emphasized by nine experts. Interviewee (I2) states: "I am thinking about the flexibility I have, because everybody has access to everything. That is a big advantage. If someone is sick for example, somebody else can temporary replace him easily".

Likewise, nine participants brought up cost reductions as a decision criterion. "But cloud-based does not automatically mean cheaper. [...] After performing a cost-benefit calculation for three years, we haven't found a cloud-based system yet that would have been cheaper [than an on premise solution]", interviewee (I5) stated. On the contrary, expert (I2) points out that "you do not have to take care about how and where to set-up servers and who you have to pay to maintain them. Also, most cloud systems are free of charge to a specific extent".

Eight participants mention the strategic impact of cloud services, e.g. regarding the increasing digitalization of processes in all industries. Hence, (I6) states: "You want to be prepared. With all the digitalization of processes in mind, it is incredible to think of what is going to happen in the next years".

The factor capital costs was mentioned by seven participants. Expert (I10) noted that "you have to be careful with pay-per-use. Until a certain break-evenpoint, it makes sense to host your own infrastructure. As for ourselves, it is currently better to work cloudbased".

Four interviewees included growth options in their decision process, mostly related to the "dynamics in terms of quick availability of resources, for example if the intensity of use increases unexpectedly" (I7).

Only two participants mentioned deferral options as a relevant influence factor. Unlike lock-in effects (see 4.2), this criterion is mainly perceived as a positive aspect using cloud systems: "The good thing is, I usually get quick access to the solution and if it does not fulfill my requirements I can terminate it just as quick" (I5).

\subsection{Organizational perspective}

The organizational perspective contains thirteen factors, whereof eleven could be detected in our data basis. Only trialability and service monitoring were not mentioned by any of the experts. The remaining factors - namely external know-how, effort expectation, complexity, organization size, voluntariness of use, roles and responsibilities, service controlling, compatibility, relative advantage, lock-in effect, and usage frequency - were emphasized by at least one of the participants.

From an organizational view, most of the decision makers $(n=8)$ in the sample valued the access to external know-how when adopting cloud services: "It just makes sense to outsource specific jobs to professional, specialized companies" (I2). In the same context, six experts emphasized the factor effort 
expectancy. Thus, interviewee (I4) stated: "We do not have the knowledge to operate such a server solution in-house, we did not acquire it and we did not even want to". Five participants additionally noted the manageable complexity of cloud systems, "especially in the early stage [of a company] since [the system] is configured within a few clicks and immediately readyto-use" (I12).

The size of the organization also matters according to five of the interviewees. For example, (I11) noted that " $a$ head of a medium-sized company acts totally different than a member of the supervisory board of a multi-corporate enterprise who calculates by the quarter and not what is going on in five years".

Three experts mentioned the fundamental willingness to use this rather new kind of technology as an important aspect. Hence, interviewee (I8) made the experience that "even in companies which intensively deal with the topic [of cloud solutions], there is a large resistance on the employee side to consistently incorporate novel technologies".

The same amount of participants $(n=3)$ mentioned the change of roles and responsibilities, which is described by participant (I8) as follows: "I think the biggest opportunity as well as the biggest risk in that respect is to not only replace the software but also to question why I do it. There is a big opportunity to work more effectively and improve cooperation".

Two interviewees noted unsolved problems regarding the service controlling when using cloudbased systems. Participant (I10) for example demanded, that "you have to be able to monitor and control who has access to your data, which means to control traffic on deep layers [of the systems]'.

Likewise, two experts highlighted the enhanced compatibility of cloud-services, especially in heterogeneous ecosystems: "The bottom line is that we have to enable access to the applications to many different users, which is relatively easy to achieve in the cloud. [...] I do not have to install a client and it does not matter if I am currently using an Android or Apple device or my Windows PC' (I8).

The factors lock-in effect and usage frequency were each mentioned by one decision maker. Interviewee (I10) stated, that " a problem is that the provider could go broke, which is a problem with Navision for example. The system does not provide backup functionality. [...] In that case, there is a lot of data only stored by your provider and if he cannot continue business, then you might have a problem".

\subsection{Environmental perspective}

The environmental perspective contains the factors energy efficiency, competitive pressure, image, subjective norm, industry characteristics, social impact and privacy.

The factor privacy was the most mentioned from the environmental perspective. All participants confirmed its relevance. Interview partner (I7) stated: "In principle, I think that the German mentality regarding privacy is reasonable. But if I am operating with cloud services I will mainly leave the pure German judicial area". He further stated that he considered the laws as "suitable to only a limited extent, conducive but undifferentiated".

Industry characteristics and social impact were seen as a relevant influence on the adoption decision by seven interviewees each. Interviewee (I11) explained the influence of the industry characteristics as follows: "We produce some parts individually for one costumer. This customer holds the exclusive user right. If I would use cloud services for the computing or storage of data, I'd run the risk of losing that asset and know-how and rather getting a contractual penalty due to the harsh policies from the customer",

An environmental aspect can be found in the factor subjective norm, which was mentioned by half of the participants. Interview partners see an obstacle in the subjective mindset of potential users. A statement of interviewee (I3) describes the problem appropriate: "[...] since we also have a lot of conservative customers, the on premise solution has a greater demand than cloud services".

Another factor of the environmental perspective is the competitive pressure. Interviewee (I8) sees difficulties with international competitors due to certain conditions in Germany and mentioned it as follows: "[...] how shall we keep up with the international competitors, when we are not able to utilize the same technology. The competitors gain competitive advantage".

The at least mentioned environmental factor is energy efficiency. Only the participant of company (I7) stated: "There are also improvements in a relatively large number of areas due to omission of environmental pollution and a more efficient usage of energy because of the digitalization".

\subsection{Individual perspective}

The individual perspective contains the factors perceived ease of use, perceived usefulness, and trust.

Trust, which means "[...] the confidence in the provider to complete his job reliably, for example regarding the processing of customer data" (I4), was emphasized by eleven of twelve interviewees. Participant (I3) additionally referred to the recent safe harbor sentence in the European Union: "The trustworthiness of providers, especially from the US, 
has suffered due to the safe harbor decision. That is why especially German companies [...] have to gain the customer's trust".

Eight participants mentioned the perceived usefulness of cloud-services as an adoption criterion, especially related to cloud-based application software (SaaS) and increased mobility: "Because I can work from everywhere, which means I am not bound to one specific workspace and able to communicate much faster with my co-workers" (I2).

Perceived ease of use eventually was brought up by three interviewees. By ease of use, the experts mostly referred to a good functionality and an easy setup process: "I think it has to be easy. When we set up cloud-services for customers, they expect an Apple-like system. You plug it in, click three times $O K$ and it works" (I6).

\subsection{New factors}

During the evaluation of the interviews, it became obvious that the literature review in section 2 does not cover all factors influencing the adoption of cloud services. In addition to the factors in table 1 , the interviews revealed five new factors: transparency (service), standardization, business model, lack of know-how and transparency (security). These factors will be shown by selected mentions in the following and further evaluated in the discussion.

Most of the decision makers $(n=10)$ in the sample value the transparency of the security. Interview partner (I10), for example, mentioned the location of the data processing and storage and stated: "The hindrances, or what can be seen as disadvantageous, are the data retention. Where is critical data geographically stored? What is with data backups? All of this is pretty important". Interviewees mainly said that they prefer more transparency concerning security and data issues.

The second most mentioned new factor is the lack of know-how. It is considered to have a negative correlation to the adoption of cloud services. Participant (I5) stated that: "If I am a SME with a lack of knowledge concerning cloud services, I would rather see it as a danger then an opportunity". That implicates that a better understanding of cloud services would improve the adoption, which was stated by more than half of the interviewees.

The business model is another newly identified factor. Interviewee (I1) recognized that the pricing model pay per use is not always suitable for microenterprises because of the unpredictable usage rate. Another participant (I5) sees a potential improvement in a decentral allocation of cloud service providers to enhance the adoption.
The second least mentioned new factor is standardization. It consists of different aspects, such as consistent general conditions, interfaces and interoperability. The least stated factor of the new factor perspective is transparency (service). One participant sees it as relevant influence for the adoption. Interviewee (I3) stated: "It has to be clearly evident for the user, who is participating in the data processing in which way".

\section{Discussion}

The following chapter contains a classification of the results derived through the empirical survey. Moreover, the new factors are arranged in the used and advanced TOE framework.

In figure 3, a subdivision of influence factors is made according to their importance.

Aspects mentioned from less than 25 percent of the interviewees can be assessed as hardly relevant, which includes seventeen influencing factors. Four factors were not mentioned by any interview partner and hence have no influence on the adoption in the underlying sample. The other thirteen factors were each mentioned from only one to three interviewees. This includes the factors transparency (service) and standardization which were not obtained in former studies.

The factors mentioned by 25 to 75 percent of interviewees are accordingly estimated to have relevant impact on the acceptance and adoption of cloud services by the investigated SMEs. This category includes fourteen factors mentioned by four to eight interviewees.

One was stated in four interviews. Three factors in each case were mentioned by five, six and eight interviewees. Four more factors can be extracted because they were mentioned in seven interviews each.

In this context, it is noteworthy that the new factors business model and lack of know-how are to be attributed to these categories of relevance. The first one was identified in six interviews. The second new factor in this category was even identified in seven interviews. It clearly shows that the newly discovered factors have a certain relevance for the influence whether to adopt cloud services or not.

Influencing factors with a high relevance for the adoption of cloud services are those mentioned in more than 75 percent of the conversations with SMEs. This involves factors stated nine times or more in different interviews. The new factor transparency (security) includes this category and was seen as an influencing factor by ten interviewees which confirms its importance. 
The individual factor trust is the third most stated. Two factors were unanimously mentioned by all interviewees. Therefore, security and privacy are the most stated. As a consequence, these factors obtained from the literature turn out to have the highest influence in the study on hand. They can be seen as the main driver or major obstacle for the adoption of cloud based solutions and services for the examined enterprises in the investigated area.

Another result of this study, beneath the classification of relevance of the influencing factors extracted from the literature, is the identification of five new factors. These are, in a descending order of relevance, transparency (service), standardization, business model, lack of know-how and transparency (security). Subsequently, we attempt to categorize these new factors in the formerly used TOEIframework.

The transparency (service) defies a classification to a particular category. Due to the interviewees, the factor consists aspects of the technological category, e.g. regarding the inspection of the procedure of data processing, as well as the organizational category, e.g. since service providers themselves shall act transparent.

However, the standardization can unequivocally be assigned to the technological category.

The third new factor, in turn, is not relatable to one particular category. It is a mélange of individual aspects, such as trust on one side and also environmental aspects, e.g. social influences or subjective norms.

Although the business model results from the technology, but it is classified as organizational. It is an organizational decision how to market a product and how to operate a value chain with it.

The fifth new influencing factor, the lack of knowhow, is firstly composed of aspects of the organizational factor complexity and on the other hand of aspects of the individual factors perceived ease of use and perceived usefulness. The technological factors, such as strategic impact and technological availability, also need to be considered

The transparency (security) is a conglomerate of factors from all categories. It combines the individual trust, the organizational service monitoring, the environmental privacy and the technological factor security. The mention of transparency (security) by ten of twelve interviewees underlines its importance and relevance as influencing factor for the adoption of cloud services.

To summarize, it can be affirmed that the new influencing factors partially build on factors form the literature, expand them or move them towards a different direction. Factors like transparency (security) are admittedly only limited classifiable within the TOEI-framework, and if so, only based on the statement of each individual interview partner.

The investigation of the factor's relevance, taking the pretest interviews into account, has revealed a notable outcome. The pretest was conducted with scientific personal with a practical focus. Hence, the statements of the pretest can be seen as scientific point of view. The interviewees in the main survey are from companies and can be seen as practical wise point of view.

During the investigation of the interviews, a significant divergence was discovered between statements from the science side in the pretest and opinions from the practice in some cases.

On one hand, there are factors ranked with a high relevance from the scientific point of view. Factors, such as transparency (service) and the lock-in-effect were mentioned by all pretest interviewees, but attracted only little to no attention by the practical side. Based on the foregoing it can be interpreted that the above mentioned factors are elaborated with a more complex mindset as it is common in the practice. It can be reasonably assumed that the science is more advanced in the area of innovation than the practice. Hence, factors like the lock-in-effect are assumed to have a potential future impact on the influence of the cloud service adoption.

On the other hand, there are factors characterized vice versa. They have a high relevance for the practical point of view but not for the scientific. This applies, e.g. for effort expectations mentioned by half of the interview partners from the main survey and from none of the pretest interviewees. This is even more significant with the factor transparency (security). The ratio between scientific and practical mention in this case is zero to ten. However, this shows the discrepancy between the both perceptions. This result can be explained by the fact that companies have a deeper insight to certain processes due to the daily use of cloud services. Moreover, the reality and theory of cloud services are not always identical. The fact that the factor transparency (security), for example, is based on individual requirements of those surveyed can be a reason that the above mentioned factor is not equally represented in both perceptions.

In case of the three most mentioned influencing factors, both views agree on security and privacy. Those were likewise stated by all fifteen interviewees. There is a small deviation in the factor trust, which was not mentioned by one interviewee from a company, concededly all remaining stated it unanimously. This additionally emphasizes the relevance of the three most stated influencing factors for the acceptance and adoption of cloud services in the examined area. 


\section{Summary and Conclusion}

The objective of the study on hand was to evaluate measures and drivers of the acceptance and adoption of cloud services in SMEs, based on a qualitative survey.

Different methods were used for this survey to provide an accurate scientific work. A rigour literature reviews was conducted and afterwards a structured interview was designed for the empirical data acquisition and aligned with the ex-ante assigned research question. The interview has been validated by the application of pretests. In the following step, the auditory recorded interviews have been transliterated and were coded according to scientific requirements.

Thirty-three factors are derived from a comprehensive literature review. Based on these factors and the data from the interviews, a frequency analysis was conducted to undertake a classification of relevance.

The study shows that the influence on the adoption is widely spread, from technological, to organizational and individually subjective assessments.

Security-related factors have been revealed as the most important ones in the surveyed area. Security, privacy and trust are the three top mentioned factors.

Furthermore, the study reveals that four factors extracted from other studies could not to be confirmed in their relevance. However, five new factors were identified and can be seen as enhancement of the already identified.

A limitation of the paper on hand is due to its research method. A quantitative clarification of the factors in a wider and broader range would lead to a more precise discussion about the influencing factors.

In summa, the study provides an interesting insight into the frame of mind towards cloud services and their adoption in the surveyed companies. Admittedly, the frequency analysis has its limitation in the dimension. Therefore, the sheer mention was analyzed but not the bipolar alignment. This limitation could be eliminated by the evaluation through a valence analysis. Thereby, the bipolarity of the statements can be taken into account and factors can be categorized by their positive or negative influence. Therefore, the results of the study on hand can be enhanced and condensed in future investigations.

Another opportunity for further research can be found in the limitation of German SMEs. Future research in this field could similarly delve into other regions and therefore identify alleged geographical characteristics of the adoption of cloud services. Although this study provides valuable insights into the decision process regarding cloud services, there are still a lot of opportunities for further investigation in this research area.

\section{References}

[1] Alshamaila, Y., Papagiannidis, S., and Li, F. Cloud computing adoption by SMEs in the north east of England: A multi-perspective framework. Journal of Enterprise Information Management 26, 3 (2013), 250-275.

[2] Asadi, S., Nilashi, M., Husin, A.R.C., and Yadegaridehkordi, E. Customers perspectives on adoption of cloud computing in banking sector. Information Technology and Management, 2016, 1-26.

[3] Benlian, A. and Hess, T. Opportunities and risks of software-as-a-service: Findings from a survey of IT executives. Decision Support Systems 52, 1 (2011), 232-246.

[4] vom Brocke, J., Simons, A., Niehaves, B., Riemer, K., Plattfaut, R., and Cleven, A. Reconstructing the Giant: On the Importance of Rigour in Documenting the Literature Search Process. 17th European Conference on Information Systems, (2009), 2206-2217.

[5] Bryman, A. and Bell, E. Business Research Methods. Oxford University Press, Oxford, 2011.

[6] Dubé, L. and Paré, G. Rigor in Information Systems Positivist Case Research: Current Practices, Trends, and Recommendations. MIS Quarterly 27, 4 (2003), 597-635.

[7] European Commission. Regulation No. 651/2014. 2014.

[8] Fabian, B., Ermakova, T., and Junghanns, P. Collaborative and secure sharing of healthcare data in multiclouds. Information Systems 48, (2015), 132-150.

[9] Gangwar, H., Date, H., and Ramaswamy, R. Understanding determinants of cloud computing adoption using an integrated TAM-TOE model. Journal of Enterprise Information Management 28, 1 (2015), 107-130.

[10] Gupta, P., Seetharaman, A., and Raj, J.R. The usage and adoption of cloud computing by small and medium businesses. International Journal of Information Management 33, 5 (2013), 861-874.

[11] Hoberg, P., Wollersheim, J., and Krcmar, H. The Business Perspective on Cloud Computing-A Literature Review of Research on Cloud Computing. AMCIS 2012 Proceedings, (2012), Paper 5.

[12] Hsu, P.F., Ray, S., and Li-Hsieh, Y.Y. Examining cloud computing adoption intention, pricing mechanism, and deployment model. International Journal of Information Management 34, 4 (2014), 474-488.

[13] KPMG and BITKOM. Cloud-Monitor 2016: Cloud Computing in Deutschland - Status quo und Perspektiven. 2016. 
[14] Lansing, J. and Sunyaev, A. Trust in cloud computing: Conceptual typology and trust-building antecedents. Data Base for Advances in Information Systems 47, 2 (2016), 5896.

[15] Lian, J.W. Critical factors for cloud based e-invoice service adoption in Taiwan: An empirical study. International Journal of Information Management 35, 1 (2015), 98-109.

[16] Lian, J.W., Yen, D.C., and Wang, Y.T. An exploratory study to understand the critical factors affecting the decision to adopt cloud computing in Taiwan hospital. International Journal of Information Management 34, 1 (2014), 28-36.

[17] Lin, A. and Chen, N.C. Cloud computing as an innovation: Percepetion, attitude, and adoption. International Journal of Information Management 32, 6 (2012), 533-540.

[18] Low, C., Chen, Y., and Wu, M. Understanding the determinants of cloud computing adoption. Industrial Management \& Data Systems 111, 7 (2011), 1006-1023.

[19] Marston, S., Li, Z., Bandyopadhyay, S., Zhang, J., and Ghalsasi, A. Cloud computing - The business perspective. Decision Support Systems 51, 1 (2011), 176-189.

[20] Mell, P. and Grance, T. The NIST Definition of Cloud Computing Recommendations of the National Institute of Standards and Technology. Nist Special Publication 145, (2011), 7.

[21] Mital, M., Pani, A.K., Damodaran, S., and Ramesh, R. Cloud based management and control system for smart communities: A practical case study. Computers in Industry 74, (2015), 162-172.

[22] Müller, S., Holm, S., and Søndergaard, J. Benefits of Cloud Computing: Literature Review in a Maturity Model Perspective. Communications of the Association for Information Systems 37, (2015), 851-878.

[23] Narasimhan, B. and Nichols, R. State of cloud applications and platforms: The cloud adopters' view. Computer 44, 3 (2011), 24-28.

[24] Oliveira, T., Thomas, M., and Espadanal, M. Assessing the determinants of cloud computing adoption: An analysis of the manufacturing and services sectors. Information and Management 51, 5 (2014), 497-510.

[25] Palvia, P., Leary, D., Mao, E., Midha, V., Pinjani, P., and Salam, a. F. Research Methodologies in MIS: An Update. Communications of the Association for Information Systems 14, 1 (2004), 526-542.
[26] Repschlaeger, J., Zarnekow, R., Wind, S., and Klaus, T. Cloud Requirement Framework: Requirements and Evaluation Criteria to adopt Cloud Solutions. 20th European Conference on Information Systems, (2012), 1-12.

[27] Rogers, E.M. Diffusion of Innovations. 2003.

[28] Sabi, H.M., Uzoka, F.M.E., Langmia, K., and Njeh, F.N. Conceptualizing a model for adoption of cloud computing in education. International Journal of Information Management 36, 2 (2016), 183-191.

[29] Sabi, H.M., Uzoka, F.M.E., Langmia, K., Njeh, F.N., and Tsuma, C.K. A cross-country model of contextual factors impacting cloud computing adoption at universities in subSaharan Africa. Information Systems Frontiers, 2017, 1-24.

[30] Schneider, S. and Sunyaev, A. Determinant factors of cloud-sourcing decisions: Reflecting on the IT outsourcing literature in the era of cloud computing. Journal of Information Technology 31, 1 (2016), 1-31.

[31] Seethamraju, R. Adoption of Software as a Service (SaaS) Enterprise Resource Planning (ERP) Systems in Small and Medium Sized Enterprises (SMEs). Information Systems Frontiers 17, 3 (2015), 475-492.

[32] Statistisches Bundesamt. Mehr als $60 \%$ der tätigen Personen in kleinen und mittleren Unternehmen. Berlin, 2015.

[33] Stieninger, M. and Nedbal, D. Diffusion and acceptance of cloud computing in SMEs: Towards a valence model of relevant factors. 2014 47th Hawaii International, (2014).

[34] Tornatzky, L.G., Fleischer, M., and Chakrabarti, A.K. The processes of technological innovation. Lexington Books, 1990.

[35] Venkatesh, V. and Davis. A Theoretical Extension of the Technology Acceptance Model: Four Longitudinal Field Studies. Management Science 46, 2 (2000), 186-204.

[36] Webster, J. and Watson, R.T. Analyzing the Past to Prepare for the Future: Writing a Literature Review. MIS Quarterly 26, 2 (2002), xiii-xxiii.

[37] Yigitbasioglu, O.M. The role of institutional pressures and top management support in the intention to adopt cloud computing solutions. Journal of Enterprise Information Management 28, 4 (2015), 579-594.

[38] Yin, R.K. Case Study Research: Design and Methods. 2009. 